\title{
ENHANCEMENT OF DISSOLUTION AND ANALGESIC ACTIVITY OF CELECOXIB USING TERNARY SYSTEM
}

\author{
Mohamed A. Amin
}

Department of Pharmaceutics and Industrial Pharmacy, Faculty of Pharmacy, Al- Azhar University, Assiut, Egypt

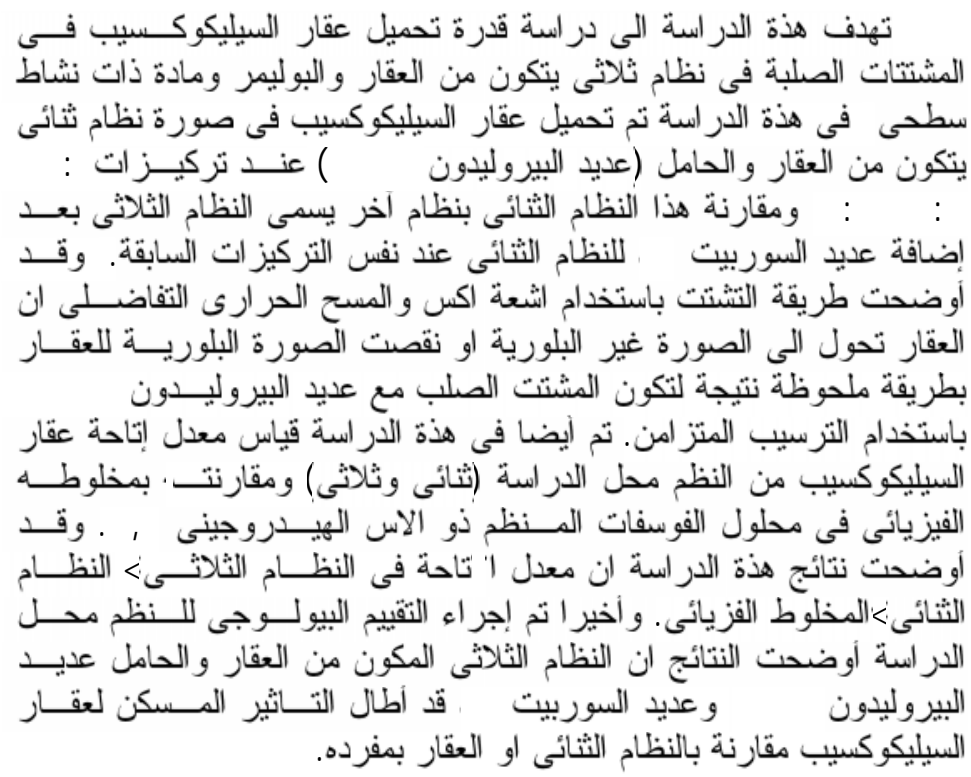

The purpose of the present study is to investigate the drug loaded solid dispersion system consisting of a drug, a carrier and a surfactant. Solid dispersions of a water insoluble drug celecoxib (CX) with PVP 40000, namely binary solid dispersion systems, was prepared at different ratios of drug to carrier [(1:1), (1:3), and (1:5)]. Polysorbate 80, a nonionic surfactant, was incorporated into the binary solid dispersion systems as a third component to obtain the ternary solid dispersion system. The solubilizing and absorption enhancement properties of ternary solid dispersion system have been investigated. The prepared solid dispersion systems (binary or ternary), at various drug- polymer ratios by mixing or co-precipitation, were characterized by differential scanning calorimetery and $X$ - ray diffractometry. The results show

Received in 19/2/2009, Received in revised form in 18/4/2009 \& Accepted in 19/4/2009 
a remarkably improved dissolution of the drug from the ternary solid dispersion systems when compared to the binary solid dispersion systems. The therapeutic activity of the ternary system was evaluated using acetic acid-induced writhing method. In-vivo experiments in mice demonstrated that the investigated ternary system (drug, polymer and surfactant) shows a greater reduction of acetic acid- induced writhing in comparison with pure drug. Moreover, the ternary system of $(\mathrm{CX})$ demonstrated antiwrithing potency 1.45 times higher than the respective binary system. Thus, the solubilizing power, the dissolution effect, and the analgesic effect were enhanced upon the addition of the investigated surfactant to the binary system of celecoxib and the polymer.

\section{INTRODUCTION}

Most of the chemical entities that are being discovered are lipophilic and have poor aqueous solubility, so they pose difficulties to the pharmaceutical scientists in their formulation. Unless a drug is delivered to its target area at a rate and concentration that minimize side effects; the drug is not beneficial to the patient and, though potentially useful, may be discarded. Among numerous ways of enhancing drug dissolution, solid dispersion of a drug in a water soluble carrier is a promising technique ${ }^{1 \& 2}$. Although the use of solid dispersions has been reported frequently in the pharmaceutical literature, still few marked products rely on the solid dispersion strategy. The main reason for this discrepancy is the possible physico-chemical instability of these structures which may be metastable $^{3 \& 4}$. Phase separation, crystal growth, or conversion from the amorphous (metastable) to the crystalline form during storage, inevitably resulted in decreased solubility and dissolution rate. However, the presence of a carrier is often adequate to prevent recrystallization $^{5 \& 6}$. The interest to use surfaceactive and self-emulsifying carriers for the solid dispersion of poorly water- soluble drugs increased in recent years ${ }^{3,7 \& 8}$. Gelucire 44/14, vitamin E, TPGs and Polysorbate 80 are examples of such carriers. Furthermore, it was reported that a solid dispersion in mixture of polyethylene glycol and Polysorbate 80 could improve the dissolution rate and enhance the bioavailability of LAB687, a poorly water soluble $\operatorname{drug}^{9}$. The bioavailability of this kind of solid dispersion showed 10-fold increase compared to the drug blend of micronized drug with microcrystalline cellulose. In addition to the solid dispersion, the system was physically and chemically stable for at least 16 months at $25^{\circ} \mathrm{C}$ and $60 \%$ RH. 
Celecoxib (Fig. 1), chemically designated as 4-[5-(4-methylphenyl)3-(trifloromethyl) - 1H- pyrazol-1-yl] benzenesulfonamide, is a specific cyclooxygenase- 2 inhibitor widely prescribed for the relief of pain and inflammation ${ }^{10}$. It inhibits the conversion of arachidonic acid to prostaglandins that mediate pain and inflammation, while it has no effect on the formation of the prostaglandins that mediates normal homeostasis in the gastrointestinal tract, kidney, and platelets $^{11 \& 12}$. It is also prescribed in the treatment of arthorpathies and adenomatous polyps, and in dentistry ${ }^{13}$. It has comparable efficacy and superior gastric tolerability ${ }^{14}$ and it is safer when compared to other non steroidal anti-inflammatory drugs ${ }^{15}$.

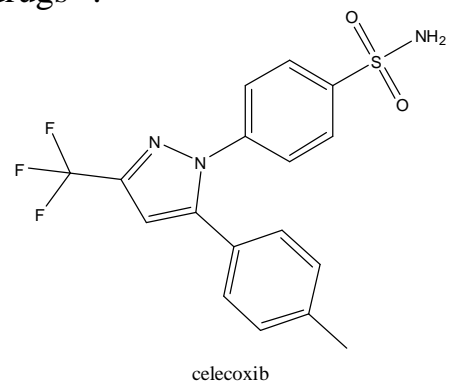

Fig. 1: Schematic structure of celecoxib

Because of its very low aqueous solubility and poor dissolution rate, celecoxib shows a large interindividual difference in bioavailability after oral administration ${ }^{16}$. Currently, several formulations are being developed to overcome the dissolution rate limiting oral absorption of celecoxib. In order to improve the stability and dissolution properties of celecoxib, a new formulation consisting of celecoxib, a hydrophilic polymer (PVP 40,000) and a surfactant (Tween 80) is used.

The selection of the polymers and the surfactants is based on the fact that one of them must dissolve the drug in the solid state, leading to a stable system without phase separation, while the other must increase the stability and dissolution rate of the drug. In the present study, PVP 40,000 was selected as a polymer because it is able to molecularly disperse celecoxib in the solid state ${ }^{17}$. Polysorbate 80 was selected as a surfactant because of its solubilizing action on several drugs ${ }^{18}$. The compatibility between the carrier and the drug seems to be a stabilizing factor for the solid dispersion. However, little has been reported on the ternary system ${ }^{19-21}$.

Therefore, the purpose of the present study is to investigate the solid state properties of both binary and ternary systems to understand how materials behave in a ternary system. Also the analgesic response of celecoxib in the ternary system was compared to the respective binary system using acetic acid induced writhing method.

\section{EXPERIMENTAL}

\section{Materials}

- Celecoxib was kindly supplied by the Egyptian international pharmaceutical industries co., (Sedico) Egypt.

- Polysorbate 80 (Tween 80) [(polyoxethylene (20) sorbiton 
monooleate (POE)] (BDH chemical Ltd. Co., Poole England).

- Polyvinyl pyrrrolidones 40,000 (sigma chemical Co., USA).

Other materials and solvents are of pharmaceutical grade and they were used without further purification.

\section{Equipment}

- Shaking water path, thermostatically controlled BF21 (SBS Instruments, Germany).

- Spectrophotometer UV.1601 (shimadzu co., Japan).

- Sieve shaker, Rx-86-1 (ColeParmer Instrument Co., USA).

- Dissolution test apparatus, SR11 6 flask (Hanson Co., USA).

- pH meter, Ama digital (Ama CO., Germany)

- X-ray diffractometer (Philips Co., Netherlands).

- T.A. 501 Differential scanning calorimeter, DSC (Shimadzu Co., Japan).

\section{Methodology}

\section{Preparation of celecoxib physical mixture}

The physical mixtures of a binary system of celecoxib with PVP 40,000 at a ratio of $(1: 1,1: 3$ and 1:5 w/w drug: carrier) were prepared by gentle, smooth blending of the calculated amounts of the drug and the carrier using a mortar and a pestle. Samples were pulverized and sieved to obtain a particle size of range of $125-250 \mu \mathrm{m}$. The obtained samples were stored in a desiccator over calcium chloride.
Preparation of celecoxib solid dispersion

Celecoxib solid dispersion of both binary and ternary systems were prepared by conventional solvent evaporation method using PVP 40,000 as a carrier. In the binary system, the solid dispersion of $1: 1$, $1: 3$ and $1: 5 \mathrm{w} / \mathrm{w}$ of drug to carrier were prepared. The mixture of drug $(100 \mathrm{mg})$ and the carrier was dissolved in methanol $(10 \mathrm{ml})$. In the ternary system, the surfactant was added into the solution of drug and the carrier to obtain the final weight ratio of drug /carrier/surfactant 1:5:1 $\mathrm{w} / \mathrm{w} / \mathrm{w}$. the solvent was evaporated under reduced pressure at $40^{\circ} \mathrm{C}$ till constant weight is obtained. The coevaporates were pulverized and were sieved to obtain a particle size range of $125-250 \mu \mathrm{m}$ and then stored in a desiccator over calcium chloride.

\section{Differential scanning calorimetery (DSC)}

Samples of the prepared systems (about $5 \mathrm{mg}$ ) were placed in aluminum pans. DSC thermograms were obtained by heating the samples from $30-250^{\circ} \mathrm{C}$ at a rate of $10^{\circ} \mathrm{C} \mathrm{min}^{-1}$ under nitrogen flow of $40 \mathrm{ml} / \mathrm{min}$.

\section{$\mathrm{X}$-ray diffraction studies}

The X-ray diffractograms for different samples with particle size range of $250-125 \mu \mathrm{m}$ were determined using Philips 1710 automated diffractometer. The relation was provided by Cuka radiation operating at $40 \mathrm{Kv}$ and current of $30 \mathrm{~mA}, \mathrm{Ka}^{-}=$ 1.5418. The system was calibrated using standard polycrystalline silicon. 
The differential patterns were achieved using continuous scan mode with $2 \theta$ ranging from $4^{\circ}$ to $60^{\circ}$. The obtained output data were represented by $2 \theta$, dA intensities and were determined via the microprocessor of the $\mathrm{PW} / 1710$.

Determination of the drug content of the prepared solid dispersions

An accurately weighed sample of the prepared solid dispersion system (binary or ternary) equivalent to 250 $\mathrm{mg}$ of the drug was added to $100 \mathrm{mg}$ volumetric flask, then dissolved in a minimum amount of ethyl alcohol and completed to volume to $100 \mathrm{ml}$ by phosphate buffer $(\mathrm{pH}=7.4)$ after suitable dilution, celecoxib content was determined spectrophotometrically at $\lambda_{\max } 247.5 \mathrm{~nm}$. Only the samples containing $100 \pm 5 \%$ of the claimed amounts of celecoxib were considered for further studies.

\section{Dissolution studies}

Dissolution experiments were carried out in triplicate using USP dissolution apparatus II with paddle at a rotation speed of $100 \mathrm{rpm}$. Powdered samples of each preparation equivalent to $25 \mathrm{mg}$ of celecoxib were added to the dissolution medium $(900 \mathrm{ml})$ of phosphate buffer, $(\mathrm{pH}=7.4)$ kept at $37 \pm 0.5^{\circ} \mathrm{C}$. At specific time intervals, $5 \mathrm{ml}$ of the dissolution medium was withdrawn and filtered through cotton plug and replaced with an equal volume of the fresh dissolution medium equilibrated at $37^{\circ} \mathrm{C}$. The samples were assayed spectrophotometrically at $\lambda_{\max } 247.5 \mathrm{~nm}$. It was found that none of investigated additives used interfered with the spectrophotometric assay of the drug in the dilution range used. The mean of three determinations was considered.

\section{In-vivo studies}

Adult mice weighing average of 25 to $35 \mathrm{~g}$ were used. The analgesic effect of celecoxib alone and some selected formulations were tested by evaluating the drug's ability to inhibit the acetic acid-induced writhing response $^{22-25}$. The mice were randomly divided into 6 groups each of 6 animals. A dose equivalent to 10 $\mathrm{mg}$ of celecoxib/ $\mathrm{kg}$ was administered orally as aqueous suspensions with $0.25 \%$ CMC (22-24). The equivalent volume $(\approx 0.5 \mathrm{ml})$ of the dose was withdrawn from stock aqueous suspension (4 mg equivalent $\mathrm{CX} / \mathrm{ml}$ ) of each tested formulation, and then ingested by the aid of an insulin syringe $100 \mathrm{IU}$ associated with a canula. An aqueous solution of $0.25 \%$ CMC $(0.5 \mathrm{ml})$ was administered orally to the control group. Afterwards, the mice were put in individual cages and, 20 minutes after drug administration, $10 \mu \mathrm{L} / \mathrm{g}$ of a $0.6 \%$ acetic acid solution was injected intrapertioneally into each one. The induced writhes were then counted for 15 minutes after acetic acid injection. For scoring purposes, a writhe is indicated by stretching of the abdomen with simultaneous stretching of at least one hind limb. The formula for computing percent inhibition is: 
$\%$ inhibition $=$ average writhes in the control group - writhe in the drug group $\mathrm{X} 100$ writhes in the control group

\section{Statistical analysis of the obtained results}

Data comparisons were performed using one-way ANOVA followed by Dunnett's post hoc test for multiple comparisons. Probability values $(\mathrm{P})$ less than 0.05 were considered to be statistically significant. The results are presented as mean \pm SEM

\section{RESULTS AND DISCUSSION}

Even though the melting method is suitable for preparing solid dispersions as drugs and ingredients melting are very easy, celecoxib physically decomposed when the temperature was raised to $\approx 160^{\circ} \mathrm{C}$. The color of celecoxib crystal gradually changed from white to yellowish color at this temperature. On the other hand, the color of the solid mass of the intact celecoxib after solvent evaporation was not changed; therefore, the solvent method was selected to prepare solid dispersions throughout this study.

\section{Differential scanning calorimetery (DSC)}

Thermal analysis, especially DSC, has proved as a powerful tool in evaluating the drug-carrier interaction $^{26}$. Interactions in the samples are derived from DSC by changes in the thermal behaviors such as elimination of an endothermic or exothermic peak or appearance of a new peak ${ }^{27}$. The thermal behavior of pure components and some selected ratio of the drug-PVP physical and dispersed mixtures are depicted in (Fig. 2). DSC curve of the pure drug shows a sharp endothermic peak at $160^{\circ} \mathrm{C}$ that indicates the anhydrous crystalline state of celecoxib. The thermal curves of PVP 40,000 show a broad peak from $57^{\circ} \mathrm{C}$ to $62^{\circ} \mathrm{C}$.

The thermal behavior of celecoxib-PVP system demonstrates the absence of interactions between celecoxib and PVP. In fact, the endothermic peak of celecoxib reduced in intensity in ratios drug: PVP 1:1 and 1:3 including physical mixture and coevaporates mixtures, as well, until fully disappearing in a ratio of 1:5 coevaporate, revealing total drug amorphization. Tween 80 was liquid at room temperature, therefore, it was not possible to record a DSC trace under the experimental conditions used. The thermal behavior of celecoxib in the ternary system was similar to that of the investigated binary systems. These results indicate that tween 80 did not play role in the thermal behavior of celecoxib. Similar findings were found by Okonogi and Puttipiatkhachron $^{28}$, who have studied the thermal behavior of binary and ternary solid dispersion systems of ofloxacin. 


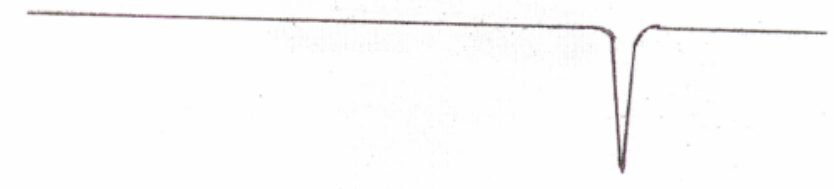

A
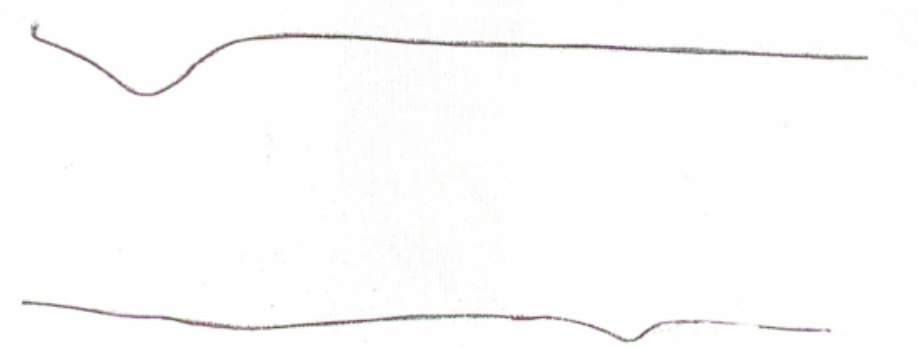

$\mathrm{C}$

$\mathrm{D}$

E

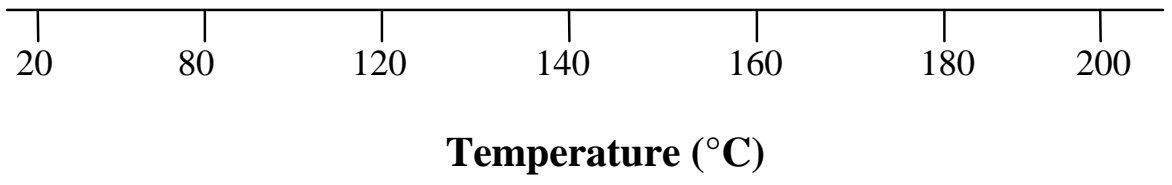

Fig. 2: DSC curves of celecoxib and various prepared systems with PVP 40,000.

A- Celecoxib alone

B- PVP 40,000 alone

C- Physical mixture (1:1)

D- Binary mixture (1:5)

E- Ternary mixture $(1: 5: 1)$ 
X-ray diffraction pattern (X-RDP)

$\mathrm{X}$-ray diffractometry patterns of celecoxib and its various ratios with PVP 40.000 are shown in (Fig. 3). The diffraction spectrum of pure celecoxib shows that the drug was crystalline in nature as demonstrated by numerous distinct peaks. The predominant peaks of the drug are observed at $2 \theta$ of $6.09^{\circ}, 11.43^{\circ}$, $15.53^{\circ}, \quad 16.82^{\circ}, \quad 22.92^{\circ}, \quad 27.72^{\circ}$, $30.31^{\circ}, 33.27^{\circ}$, and $35.66^{\circ}$ etc. (Fig. 3 trace A). PVP 40,000 shows no characteristic peaks indicating its amorphous nature. (Fig. 3, trace B). Physical mixture of the drug with PVP 40,000 at ratio $(1: 5 \mathrm{w} / \mathrm{w}$ drug: carrier) still show the characteristic peaks of the drug.

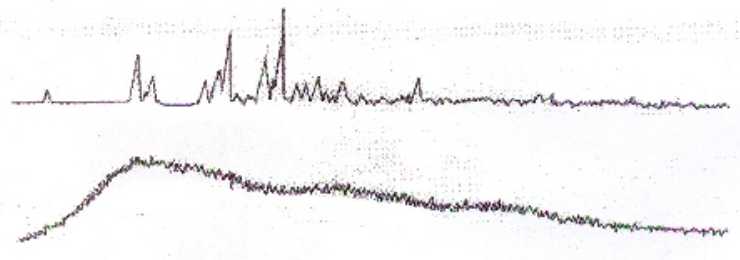

A

B

C

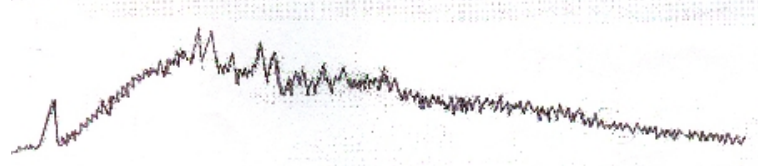

D

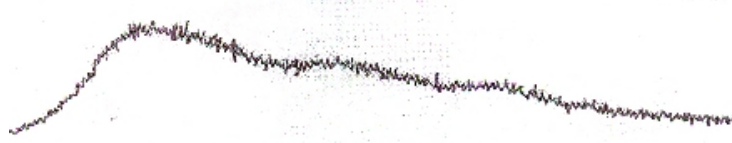

E

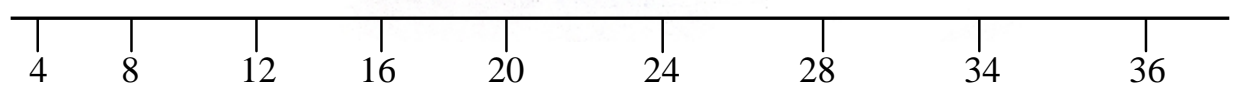

$2 \theta$

Fig. 3: XRD spectra of celecoxib and various prepared systems with PVP 40,000
A-Celecoxib alone
C-Physical mixture (1:1)

B-PVP 40,000 alone

D-Binary mixture (1:5)

E-Ternary mixture (1:5:1) 
The X-RD pattern of celecoxib binary solid dispersions with PVP at 1:5 ratios shows the decrease of the peak intensity of celecoxib. The XRD patterns of celecoxib ternary solid dispersions were similar to those of binary system. Incorporation of tween 80 had no effect on XRD pattern of celecoxib in the solid dispersion. It was considered that Tween 80 might exist in the amorphous region of both celecoxib and PVP. Similar findings have been found by Okonogi and Puttipiatkhachron $^{28}$, who have found that Tween 80 was incorporated into the amorphous region of the polymer. These results were similar to that obtained by DSC studies.

\section{In-vitro dissolution studies}

The dissolution of celecoxib from the prepared celecoxib binary and ternary solid dispersions with PVP 40,000 in comparison with the intact drug is illustrated in (Figs. 4\&5). It is clear that the rate of dissolution of pure celecoxib in $\mathrm{pH} 7.4$ is very low, less than $4 \%$ of the drug being dissolved within 2 hrs. In all cases, solid dispersions of CX with PVP 40,000 exhibit faster dissolution rates than that of the intact drug. This may be due to the effect of molecular dispersion of the drug in PVP 40,000 and the effect of Tween 80 in the ternary system.

The dissolution rate of celecoxib from all the prepared systems at the same ratios of the drug with PVP 40,000 decreased in the following order:
Ternary system > binary system > physical mixture $>$ drug alone

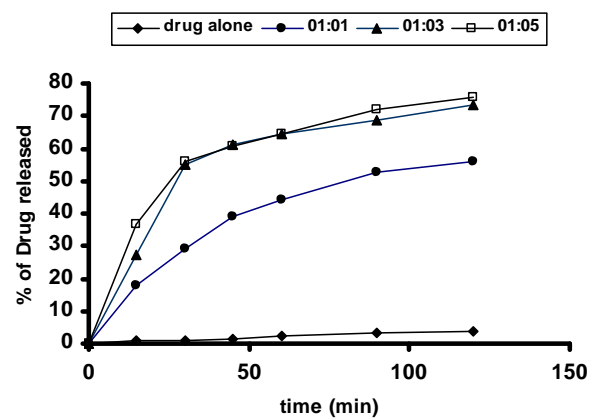

Fig. 4: Dissolution profile of celecoxib and its physical mixtures with PVP 40,000

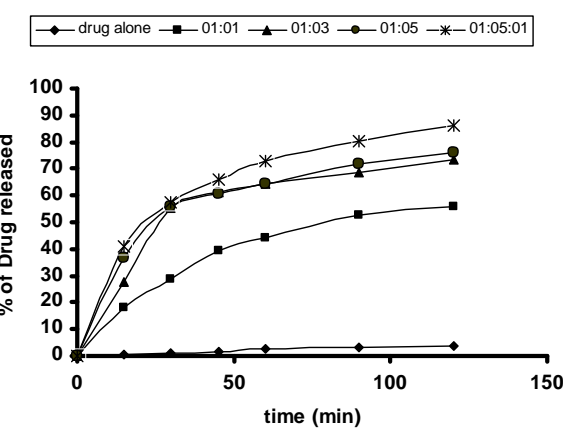

Fig. 5: Dissolution profile of celecoxib and its co-evaporates with PVP 40,000

The remarkable improved of the dissolution rate of celecoxib from its binary system at a ratio of $(1: 5 \mathrm{w} / \mathrm{w}$ drug: PVP) can be attributed to the existence of the drug in a reduced crystalline pattern as illustrated by DSC and X-ray diffraction studies. Also, PVPs have good water solubility and can improve the wettability of the dispersed compound. Similar results ${ }^{29}$ have been reported for a series of NSAIDS 
including mefenamic acid, glafenin and flufenamic acid in co-evaporates with PVP.

In the prepared ternary system, the dissolution results exhibit the fastest at the same ratio of the polymer. Upon exposure to the dissolution medium, Tween 80 was oriented onto the surface of celecoxib particles and decreased drug surface tension. This led to the extremely fast interaction of drug with Tween 80 and increased drug wettability ${ }^{28}$. It is noticed that the concentration of Tween 80 in the solid dispersion is high enough to make the diffusion layer surrounding solid dispersion surface in the dissolution medium higher than its critical micelle concentration. Therefore, it was considered that the hydrophobic portion of celecoxib was also solubilized via surfactant micelles. These effects of Tween 80 and the desirable polymer of PVP, in addition to the suitable proportional drug/carrier/ surfactant of 1:5:1 w/w/w gave extremely predominant increment dissolution of celecoxib. Dissolution rate constants $\left(\mathrm{k}_{1}\right)$ were calculated from the slopes of the firstorder linear plots of the dissolution data. Dissolution efficiency $\left(\mathrm{DE}_{30}\right)$ values based on the dissolution data were calculated as done by $\mathrm{Khan}^{30}$, the dissolution parameters were summarized in (Table 1). As can be seen, PVP 40,000 was effective in enhancing the drug dissolution performance, and its efficacy depended on its content in the physical mixture and the system preparation method. All solid dispersion systems either (binary or ternary) exhibited higher rates of dissolution and dissolution efficiency values than drug alone. The $\mathrm{k}_{1}$ and $\mathrm{DE}_{30}$ values were increased upon the addition of Tween 80 (ternary system). The ternary system [drug/carrier/surfactant of 1:5:1 $\mathrm{w} / \mathrm{w} / \mathrm{w}$ ] yields a 63.62 fold increase in the dissolution rate of celecoxib, whereas the binary system [drug/ carrier 1:5 w/w] yields 33.14 increase in the dissolution rate.

\section{In-vivo experiments}

The amorphous dispersed binary system at a ratio of $1: 5 \mathrm{w} / \mathrm{w}$ drugs to polymer and the ternary system of 1:5:1 drug: Polymer: surfactant were selected for in-vivo experiments in mice. The results of writhing test were presented in (Table 2). These results demonstrate that the analgesic activity of celecoxib was significantly potentiated when the drug was formulated in solid dispersions with PVP 40,000. Also, the effects of Tween 80 at a suitable ratio of drug/carrier/ surfactant of 1:5:1 gave extremely analgesic effect when compared with binary system or drug alone. This obvious enhancement was also considered to be due to the solubilizing effect of Tween 80 existing in the solid dispersion system associated with carrier. These results were in agreement with the findings of Chowdany and Srinivas ${ }^{31}$ who have studied the influence of hydrophilic polymer on celecoxib complexation with HP $\beta C D$. They have found that solid inclusion complexes of HP $\beta C D$ with hydrophilic polymers yielded rates of dissolution and hence analgesic response several times higher than that of celecoxib of its complexes with $\mathrm{HP} \beta C D$. 
Table 1: Dissolution parameters of different celecoxib formulation.

\begin{tabular}{||c|c|c|c|c|c||}
\hline system & $\begin{array}{c}\text { Preparation } \\
\text { techniques }\end{array}$ & $\begin{array}{c}\% \text { dissolved } \\
\text { in } 10 \text { minutes }\end{array}$ & $\mathrm{DE}_{30} \%$ & $\mathrm{~K}_{1}(\mathrm{~min})^{-1}$ & $\begin{array}{c}\text { Increase in } \\
\mathrm{K}_{1}\left(\text { folds }^{+}\right.\end{array}$ \\
\hline Celecoxib (CX) & Drug alone & $0.56 \pm 1.05$ & 7.51 & 0.0089 & - \\
\hline $\begin{array}{c}\text { CX-PVP 40,000 } \\
(1: 5)\end{array}$ & Physical mixture & $13.01 \pm 1.37$ & 22.83 & 0.1372 & 15.14 \\
\hline $\begin{array}{c}\text { CX-PVP 40,000 } \\
(1: 5)\end{array}$ & $\begin{array}{c}\text { Co-evaporates } \\
\text { Binary system }\end{array}$ & $35.60 \pm 1.92$ & 28.65 & 0.2951 & 33.14 \\
\hline $\begin{array}{c}\text { CX-PVP 40,000- } \\
\text { Tween } \\
(1: 5: 1)\end{array}$ & $\begin{array}{c}\text { Co-evaporates } \\
\text { Ternary system }\end{array}$ & $39.10 \pm 1.25$ & 56.41 & 0.5664 & 63.62 \\
\hline
\end{tabular}

$\mathrm{DE}_{30} \%$ : Dissolution efficiency

Table 2: Analgesic activity of different celecoxib formulation.

\begin{tabular}{|c|c|c|c|}
\hline System & $\begin{array}{c}\text { Drug: carrier: } \\
\text { surfactant ratio w/w/w }\end{array}$ & $\begin{array}{c}\text { \#Writhes } \\
\text { Number }\end{array}$ & $\begin{array}{c}\text { Inhibition } \\
\%\end{array}$ \\
\hline Control & $0: 0: 0$ & $65.8 \pm 6.6$ & -- \\
\hline Drug alone (CX) & $1: 0: 0$ & $45.0 \pm 2.5$ & 22.66 \\
\hline $\begin{array}{c}\text { Binary system } \\
\text { CX-PVP 40,000 (1:5) }\end{array}$ & $1: 5: 0$ & $21.12 \pm 12 *$ & 66.20 \\
\hline $\begin{array}{c}\text { Ternary system } \\
\text { CX-PVP 40,000-Tween } \\
(1: 5: 1)\end{array}$ & $1: 5: 1$ & $5.11 \pm 11 * *$ & 96.50 \\
\hline
\end{tabular}

\# Values are expressed as mean \pm SEM. The experiments were carried out three times at room temperature to confirm the results. Data comparisons were performed using one-way ANOVA followed by Dunnett's test. $* \mathrm{P}<0.05$, ** $\mathrm{P}<0.01$, as compared with control.

\section{Conclusion}

The binary solid dispersion of the drug with PVP 40,000 exhibited faster dissolution rate than that of the drug alone. The enhancement in the drug-dissolution rate was obtained by the ternary solid dispersion system using Tween 80 as a third component. Tween 80 plays an important role in drug dissolution from the ternary system by improving drug molecular dispersion in the polymer matrix as well as drug wettability and solubility. The results of in-vivo experiments in mice correlate well with the results of in-vitro dissolution.

\section{REFERENCES}

1- WL. Chiou and S. Riegelman, J. Pharm. Sci., 60, 1281 (1971).

2- DQM. Craig, Int. J. Pharm., 231, 131 (2002). 
3- A.T. M. Serajuddin, J. Pharm. Sci., 88,1058 (1999).

4- G. Leuner and Dressman, Eur. J. Pharm. Biopharm., 50, 47 (2000).

5- T. Motsumoto and G. Zografi, Pharm. Res., 61, 1722 (1999).

6- G. Vanden Mooter, M. Wuyts, R. Blaton Basson, P. Grobet., P. Augustijins and R. Kinget, Eur. J. Pharm. Sci., 12, 261 (2001).

7- A. T. M. Serajuddin, P. C. Sheen and M. A. Augustine, J. Pharm. Sci., 79, 463 (1990).

8- K. R. Morris, G. T Knipp and A. T. M. Serajuddin, ibid., 81, 1185 (1992).

9- R. H. Dannenfelser, S. Bateman and A. T. M. Serajuddin, ibid., 93, 1165 (2004).

10- G. S. Geis, Scand J. Rheumatol. Suppl., 28, 31 (1999).

11- J. Fort, Am. J. Orthop., 28, 13 (1999).

12- N. M. Davies, T. W. Gudde and H. A. Dekeuw, Expert Opin Pharmacother., 2, 139 (2001).

13- P. A. Moore and E. V Hersh, J. Am. Den. Assoc., 132, 451 (2001).

14- E. Tindall, J. Am. Osteopath. Associ., 99, 513 (1999).

15- M. Dougados, J. M. Beier and I. Joichine, Arthritis Rheum., 44, 180 (2001).

16- W. S. David, In "Physician's Desk Reference", $56^{\text {th }}$ ed., Oxford, England, 2002, pp. 2676-2680.

17- G. H. Afrasiabi, F. Sadeghi and A. Ghazi, Drug Dev. Ind. Pharm., 29, 173 (2003).

18- L.Yu-Li, J. Control. Rel., 90, 37 (2003).
19- C. Norman, A. Taakko, S. Dorothy and R. Thomas, Eur. J. Pharm. Biopharm., 71, 47 (2009).

20- W. Xin, M. Armand and M. Guy, Int. J. Pharm., 303, 54 (2009).

21- K. Verreck, G. Peeters, M. Brewester and G. Van den Mooter, Eur. J. Pharm. Biopharm., 72, 38 (2009).

22- R. Tanaka and T. Shimotori, Arzneim Forsch., 42, 935 (1992).

23- M. Inouek, H. Fujisawa and A. Motonago, Biopharm. Bull., 17, 1577 (1994).

24- N. A. Adhage and P.R Vavia, Pharm. Pharmacol. Commun., 6, 13 (2000).

25- N. Zerrouk, N. Mennini, M. Francesca, C. Chemtob and P. Mura, Eur. J. Pharm. Biopharm., 57, 93 (2004).

26- S. A. Nour, Bull. Fac. Pharm. Cairo Univ., 31, 219 (1993).

27- J. L. Ford and P. Timmins, In "Pharmaceutical Thermal Analysis, Techniques and Applications", Ellis Horwood limited, 1989, pp. 155, 177, 239.

28- S. Okonogi and S. Puttipiatkhachron, Pharm. Sci. Tech., 7, 52, (2006).

29- P. E. Luner, S. R. Babu and S. C. Mehta, Int. J. Pharm., 128, 29 (1996).

30- K. A. Khan, J. Pharm. Pharmcol., 27, 48 (1975).

31- K. P. R. Chowdary and S. V. Srinivas, Pharm. Sci. Tech., 7, 79, (2006). 